\title{
Relationship between Gene Polymorphisms and Urine lodine Levels on Susceptibility to Thyroid Peroxidase Antibody Positivity in the Chinese Population
}

\author{
Bing Han Chi Chen YiChen Ningjian Wang Jie Yu Jing Cheng \\ Yingchao Chen Chunfang Zhu Yingli Lu \\ Institute and Department of Endocrinology and Metabolism, Shanghai Ninth People's Hospital Affiliated to \\ Shanghai Jiaotong University School of Medicine, Shanghai, China
}

\section{Keywords}

SNP · Interaction · Chinese $\cdot$ Thyroid peroxidase antibodies · lodine $\cdot$ Autoimmune thyroid disease

\begin{abstract}
Background/Aims: Hashimoto thyroiditis, characterized by positive thyroid peroxidase antibodies (TPOAbs), is caused by the interaction of genetic and environment factors. The aim of this study was to clarify the interaction of gene polymorphisms and iodine intake in the incidence of TPOAb positivity. Methods: 1,733 subjects were included in this study. Genomic DNA was extracted from peripheral blood white cells. Four SNPs (rs11675434 [TPO], rs3094228 [HCP5], rs9277555 [HLA-DPB1], and rs301799 [RERE]) were selected for genotyping. Weighted TPOAb genetic risk score (GRS) was calculated based on these 4 SNPs. Thyroid hormones and autoimmune antibodies (TPOAb and thyroglobulin antibody) were determined using the electrochemiluminescence immunoassay method. Results: The mean serum thyrotropin level in TPOAb-positive subjects was higher than in TPOAb-negative subjects $(p<0.01)$. Genotype GG of rs9277555 was associated with an increased risk of TPOAb positivity (OR $=1.64,5-95 \% \mathrm{Cl} 1.09,2.47, p=0.02)$. Genotype TT of rs11675434 showed marginal increased risk of TPOAb positivity $(\mathrm{OR}=1.57,5-95 \% \mathrm{Cl} 1.01,2.43, p=0.048)$. Logistic
\end{abstract}

regression analysis showed TPOAb-GRS and rs9277555 were associated with TPOAb positivity (OR $=5.09,5-95 \% \mathrm{Cl} 1.30$, $19.91, p=0.02$ and $\mathrm{OR}=1.30,5-95 \% \mathrm{Cl} 1.05,1.61, p=0.02)$. Subjects with a high TPOAb-GRS had a $52 \%$ increased risk of TPOAb positivity compared to subjects with a low TPOAbGRS (OR 1.52, 5-95\% Cl 1.05, 2.21, $p=0.03$ ). Conclusion: TPOAb-GRS was associated with an increased risk of TPOAb positivity in a Chinese Han population. This effect might be attribute to rs9277555.

(C) 2020 European Thyroid Association Published by S. Karger AG, Basel

\section{Background}

Autoimmune thyroid disease (AITD), including autoimmune hypothyroidism (Hashimoto thyroiditis) and autoimmune hyperthyroidism (Graves' disease), affects $2-5 \%$ of the general population. Its risk factors included genetic susceptibility and environmental factors $[1,2]$. Thyroid peroxidase antibody (TPOAb) concentrations are elevated in more than $90 \%$ patients with Hashimoto thyroiditis $[3,4]$. Furthermore, TPOAb is mostly related to heritability [5]. It was estimated that genetic factors ac-

Bing Han and Chi Chen contributed equally to the manuscript.
Yingli $\mathrm{Lu}, \mathrm{MD}, \mathrm{PhD}$ and Bing $\mathrm{Han}, \mathrm{MD}, \mathrm{PhD}$

Institute and Department of Endocrinology and Metabolism Shanghai Ninth People's Hospital Affiliated to Shanghai Jiaotong University School of Medicine, Shanghai 200011 (China)

luyingli2008@126.com and hanbing1423@163.com 
counted for $73 \%$ of TPOAb positivity as well as $61 \%$ males and $72 \%$ females on serum TPOAb concentrations [6]. However, the possible causative genes of TPOAb remain unknown. In a previous study, significant associations were detected at TPO (rs11675434), ATXN2 (rs653178), and BACH2 (rs10944479) for TPOAb positivity, as were associations between TPO (rs11675434), MAGI3 (rs1230666), and KALRN (rs2010099) and TPOAb levels [2]. Using genome-wide association data, Schultheiss et al. [1] found four novel SNPs (RERE rs301799, HCP5 rs3094228, HLA-DOB rs1894407, HLA-DPB1 rs9277555) and five known genetic loci (MAGI3 rs1230666, BACH2 rs10944479, TPO rs11675434, ATXN2 rs653178, KALRN rs2010099) were associated with TPOAb.

Insufficient iodine intake can cause goiter, while more than adequate iodine intake can also result in thyroid disorders. In 1996, a mandatory universal salt iodization program was performed. Since then, China has eliminated iodine deficiency disorders, and iodine status has been determined to be more than adequate [7]. In another survey reported by the institute of iodine deficiency disorders, national median urine iodine concentration was $238.6,184.4$, and $174.4 \mu \mathrm{g} / \mathrm{L}$ in children, pregnant women, and lactating women, respectively. The urinary iodine level of children was higher than an adequate level, while both pregnant women and lactating women were at an adequate level [8]. Shan et al. [9] performed a large crosssectional study to investigate the correlation of iodine status with thyroid disorder. In cities with more than adequate iodine intake, the prevalence of positive TPOAb and thyroglobulin antibody (TGAb) as well as overt hypothyroidism and subclinical hypothyroidism was higher than the prevalence in adequate iodine intake cities [9].

In this study, we compared the thyroid hormone levels between TPOAb-positive and -negative groups in the general population who live in an iodine-replete area of China. Furthermore, TPOAb genetic risk score (GRS) was calculated and regression analysis was used to study the risk factors of TPOAb positivity.

\section{Material and Methods}

\section{Subjects}

The data was from the SPECT-China study, a cross-sectional investigation of the prevalence of metabolic diseases and risk factors in East China [10]. In May 2016, a total of 2,225 residents were recruited from 12 communities in Huangpu district, which is located in downtown Shanghai and has a population of 909,000 permanent residents [11]. Residents with missing urine iodine data $(n=280)$, thyroid operation $(n=53)$ or subacute thyroiditis $(n=$ $1)$ in ultrasound, self-reported thyroid operation $(n=8)$ or hyper-

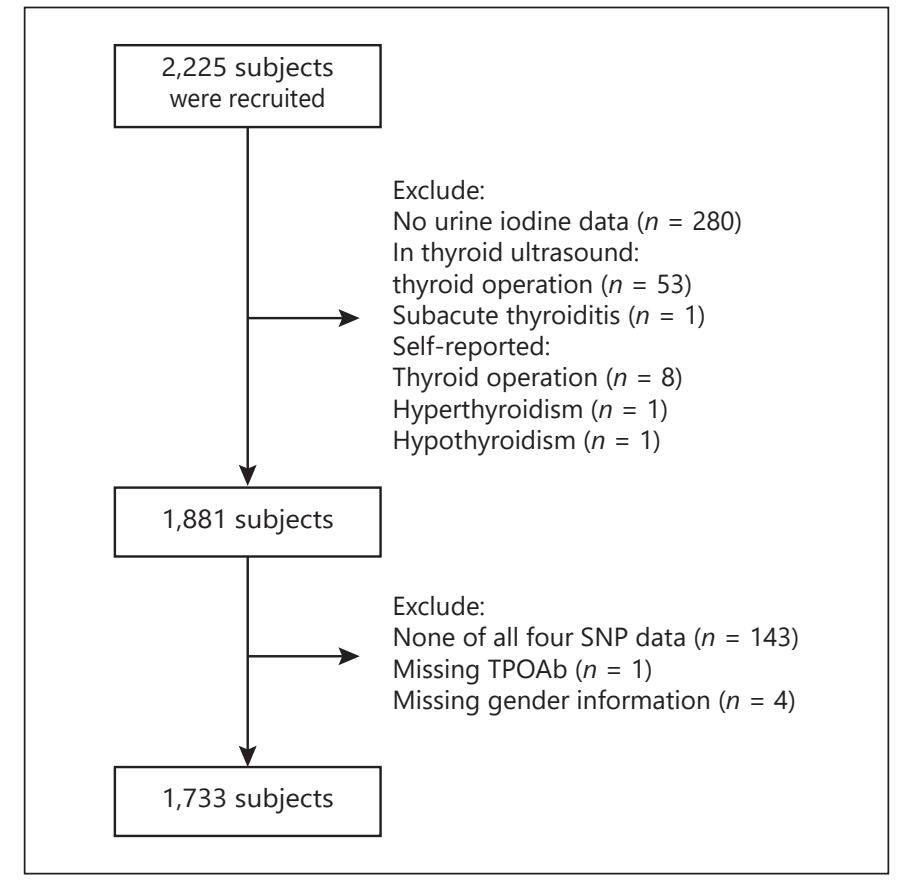

Fig. 1. Flowchart of this study.

thyroidism/Graves' disease $(n=1)$ or hypothyroidism/Hashimoto thyroiditis $(n=1)$, none of four SNPs data $(n=143)$, or without TPOAb $(n=1)$ or gender information $(n=4)$ were excluded. Finally, this study included a total of 1,733 subjects (Fig. 1). Moreover, we compared age, sex, waist circumference (WC), smoking, and TPOAb positivity between 280 subjects without urine iodine data and finally included 1,733 subjects. There was no significant difference in WC ( $p=0.999)$, TPOAb positivity $(p=0.531)$, and sex $(p=0.191)$ between these two groups. However, there was significant difference in smoking $(p=0.018)$ and age $(p<0.001)$ between two groups. The study was approved by the Ethics Committee of Shanghai Ninth People's Hospital, Shanghai Jiao Tong University School of Medicine and was performed in accordance with the Declaration of Helsinki. All the participants signed written informed consent forms before data collection.

\section{Genomic DNA Extraction and Genotyping}

Seven SNPs were selected according to previous literature [1, $2]$, including rs10944479, rs11675434, rs1230666, rs3094228, rs653178, rs9277555, and rs301799. DNA was extracted from peripheral white blood cells with a DNA Blood Mini Kit (DP603; Tiangen Biotech Co., Ltd., Beijing, China) on an automated nucleic acid extraction instrument (YOSE-S32; Tiangen Biotech Co., Ltd.). Specific assays were designed with the Geneious Pro (version 4.8.3; https://www.geneious.com/). Mass determination was carried out with the JUNO, and data acquisition was performed by Fluidigm SNP Genotyping Analysis version 4.1.3 software (Fluidigm Corporation, South San Francisco, CA, USA). The call rates of all SNPs were 98\%. rs10944479, rs653178, and rs1230666 had only one allele; therefore, these three SNPs were excluded. Finally, rs9277555 (HLA-DPB1, chr6:33087828), rs11675434 (TPO, chr2: 1404043), rs301799 (RERE, chr1:8429242), and rs3094228 (HCP5,
80

Eur Thyroid J 2021;10:79-85

DOI: $10.1159 / 000506701$
Han/Chen/Chen/Wang/Yu/Cheng/Chen/ $\mathrm{Zhu} / \mathrm{Lu}$ 
Table 1. Comparison of TPOAb-negative and -positive groups in different demographic characteristics

\begin{tabular}{lccc}
\hline Variables & TPOAb- & TPOAb & $p$ values \\
\hline Males, $n(\%)$ & $580(38.4)$ & $49(22.1)$ & $<0.001$ \\
Females, $n(\%)$ & $931(61.6)$ & $173(77.9)$ & $<0.001$ \\
Age, years & $60.65 \pm 11.14$ & $61.27 \pm 9.63$ & 0.44 \\
TSH, mIU/L & $1.95(1.40-2.71)$ & $2.58(1.66-3.80)$ & $<0.001$ \\
TT3, nmol/L & $1.72(1.56-1.91)$ & $1.75(1.56-1.93)$ & 0.39 \\
TT4, nmol/L & $112.20(100.10-124.50)$ & $112.65(97.98-127.70)$ & 0.84 \\
FT3, pmol/L & $5.07(4.74-5.39)$ & $4.94(4.63-5.28)$ & 0.002 \\
FT4, pmol/L & $16.13(15.05-17.60)$ & $15.73(14.57-17.31)$ & 0.05 \\
Urine iodine, $\mu \mathrm{g} / \mathrm{L}$ & $104.84(70.40-156.25)$ & $98.98(69.29-161.80)$ & 0.57 \\
Blood iodine, $\mu \mathrm{g} / \mathrm{L}$ & $60.41(44.09-81.37)$ & $60.33(44.77-82.70)$ & 0.60 \\
WC, cm & $82.73 \pm 9.81$ & $81.53 \pm 9.25$ & 0.09 \\
Smoking, $\%$ & 16.6 & 12.3 & 0.04 \\
\hline
\end{tabular}

TSH, serum thyrotropin; TT3, triiodothyronine; TT4, thyroxin; FT3, free triiodothyronine; FT4, free thyroxin; WC, waist circumference.

chr6:31462150) were included in the study. These four SNPs were in hardy Weinberg equilibrium. Although rs9277555 and rs3094228 were located in chromosome 6, there was no linkage disequilibrium relationship $\left(r^{2}=0\right)$ between these two SNPs. The nucleotide sequence of primers and description for the 4 SNPs are shown in online supplementary Tables 1 and 2 (see www. karger.com/doi/10.1159/000506701).

\section{Measurements}

Demographic information including lifestyle risk factors and personal thyroid disease were obtained from standardized questionnaires administered by trained staff from Shanghai Ninth People's Hospital. Current smoking was defined as having smoked at least 100 cigarettes in one's lifetime and currently smoking cigarettes [12]. WC was measured at a level midway between the lowest rib and the iliac crest. Spot urine and blood specimens were collected from each participant in the morning after an overnight fast. Concentrations of serum thyrotropin, TPOAb, and TGAb, thyroxin, free thyroxin, triiodothyronine, and free triiodothyronine levels were determined using the electrochemiluminescence immunoassay method (SIEMENS immulite 2000, Germany). TPOAb higher than $60 \mathrm{IU} / \mathrm{ml}$ was defined as serum TPOAb positivity. Urine iodine concentration and serum iodine were determined with arsenic cerium catalytic spectrophotometry (WS/T 107.1-2016) and inductively coupled plasma-mass spectrometer (ICP-MS), respectively, as previously described [11].

\section{Genetic Risk Score Calculation and Statistical Analysis}

The additive genetic model for each SNP (coded as 0-2) was used to construct GRS, which was calculated as the sum of the number of TPOAb positivity risk alleles at each locus multiplied by the respective $\beta$ coefficient reported by the previously published meta-analysis [2].

Statistical analysis was carried out using SPSS 22.0 (IBM Corporation, Armonk, NY, USA). General characteristics are summarized as means \pm SD or median with the interquartile range for continuous variables and as proportion for categorical variables. Student's $t$ test was used for comparison of normally distributed data. Mann-Whit- ney $\mathrm{U}$ test and the Kruskal-Wallis test were used for comparison of two groups in case of non-normally distributed data. The significant difference between two groups was analyzed by the chi-squared test for percentages calculated for categorical variables.

Logistic regression analyses were used to investigate the association of TPOAb positivity and other risk factors. The GRS and urine iodine interactions were evaluated by putting GRS (continuous), urine iodine (continuous), and the multiplicative interaction term (GRS $\times$ urine iodine) in the same multivariable logistic regression model [13]. The results are expressed as odds ratio (OR) and 5-95\% confidence intervals (CIs). The presence of thyroid autoantibodies was associated with gender and increasing age [14]. In 2019, a cross-sectional study demonstrated that regular smoking was associated with decreased concentrations of TPOAb $(\beta=$ -0.287 ) [15]. Chen et al. [16] found TPO/TGAb positivity was positively associated with WC in both genders $(p=0.020$ in men; $p<$ 0.001 in women). Thus, we adjusted age (continuous), sex, smoking, and WC (continuous) in logistic regression analysis. All reported $p$ values were two-tailed, and those less than 0.05 were considered statistically significant.

\section{Results}

Study Population and Thyroid Marker Characteristics 1,733 subjects (age $60.7 \pm 11.0$ years) were selected, including 629 men (age $61.8 \pm 11.4$ years) and 1,104 women (age 60.1 \pm 10.7 years). In Table 1, the percentage of males in TPOAb-positive group was higher than TPOAb-negative group (38.4 vs. $22.1 \%, p<0.001$ ). Serum thyrotropin in TPOAb-positive subjects was higher than in TPOAbnegative subjects $(p<0.001)$. There was no significant difference in blood or urine iodine in TPOAb-positive group compared to the TPOAb-negative group (98.98 vs. 104.86 for urine; 60.33 vs. 60.41 for blood). 
Table 2. Genetic risk estimation for 4 SNPs and TPOAb

\begin{tabular}{|c|c|c|c|c|c|}
\hline \multirow[t]{2}{*}{ SNP } & \multirow{2}{*}{$\begin{array}{l}\text { Genotypes } \\
\text { or alleles }\end{array}$} & \multicolumn{2}{|c|}{ Frequencies, $n(\%)$} & \multirow[t]{2}{*}{ OR (5-95\% CI) } & \multirow[t]{2}{*}{$p$ value } \\
\hline & & TPOAb- & $\mathrm{TPOAb}+$ & & \\
\hline \multirow[t]{5}{*}{ rs9277555 } & $\mathrm{AA}$ & $395(26.2)$ & $45(20.3)$ & 1 & - \\
\hline & $\mathrm{AG}$ & $783(51.8)$ & $115(51.8)$ & $1.29(0.90,1.86)$ & 0.17 \\
\hline & GG & $332(22.0)$ & $62(27.9)$ & $1.64(1.09,2.47)$ & 0.02 \\
\hline & $\mathrm{A}$ & 1,573 & 205 & 1 & - \\
\hline & G & 1,447 & 239 & $1.27(1.04,1.55)$ & 0.02 \\
\hline \multirow[t]{5}{*}{ rs11675434 } & $\mathrm{CC}$ & $719(47.6)$ & $101(45.5)$ & 1 & - \\
\hline & $\mathrm{CT}$ & $651(43.1)$ & $90(40.5)$ & $0.98(0.73,1.33)$ & 0.92 \\
\hline & TT & $141(9.3)$ & $31(14.0)$ & $1.57(1.01,2.43)$ & 0.048 \\
\hline & $\mathrm{C}$ & 2,089 & 292 & 1 & - \\
\hline & $\mathrm{T}$ & 933 & 152 & $1.17(0.94,1.44)$ & 0.15 \\
\hline \multirow[t]{5}{*}{ rs301799 } & $\mathrm{CC}$ & $47(3.1)$ & $6(2.7)$ & 1 & - \\
\hline & $\mathrm{CT}$ & $426(28.2)$ & $65(29.3)$ & $1.20(0.49,2.91)$ & 0.70 \\
\hline & TT & $1,038(68.7)$ & $151(68.0)$ & $1.14(0.48,2.71)$ & 0.77 \\
\hline & $\mathrm{C}$ & 520 & 77 & 1 & - \\
\hline & $\mathrm{T}$ & 2,502 & 367 & $0.99(0.76,1.29)$ & 0.94 \\
\hline \multirow[t]{5}{*}{ rs3094228 } & $\mathrm{AA}$ & $1,104(73.1)$ & $159(71.6)$ & 1 & - \\
\hline & $\mathrm{AG}$ & $367(24.3)$ & $56(25.2)$ & $1.06(0.76,1.47)$ & 0.73 \\
\hline & GG & $39(2.6)$ & $7(3.2)$ & $1.25(0.55,2.83)$ & 0.60 \\
\hline & $\mathrm{A}$ & 2,575 & 374 & 1 & - \\
\hline & G & 445 & 70 & $1.08(0.82,1.43)$ & 0.57 \\
\hline
\end{tabular}

Table 3. Association of TPOAb positivity (dependent factor) and other factors by logistic regression analysis

\begin{tabular}{llc}
\hline & OR $(5-95 \%$ CI $)$ & $p$ value \\
\hline rs9277555 & $1.30(1.05,1.61)$ & 0.02 \\
rs11675434 & $1.15(0.93,1.42)$ & 0.20 \\
rs301799 & $0.97(0.74,1.28)$ & 0.84 \\
rs3094228 & $1.04(0.79,1.39)$ & 0.76 \\
GRS $^{\dagger}$ & $5.09(1.30,19.91)$ & 0.02 \\
Sex & $2.53(1.71,3.74)$ & $<0.001$ \\
Age & $1.01(1.00,1.03)$ & 0.15 \\
Smoking & $0.69(0.46,1.04)$ & 0.08 \\
WC & $1.00(0.98,1.02)$ & 0.85 \\
Urine iodine & $1.00(1.00,1.00)$ & 0.27 \\
GRS $\times$ urine iodine & $1.00(1.00,1.01)$ & 0.76 \\
GRS $\times$ smoking & $0.19(0.01,5.11)$ & 0.32 \\
GRS $\times$ sex & $0.20(0.01,4.70)$ & 0.31 \\
\hline
\end{tabular}

It was adjusted by 4 SNPs, sex, age, smoking, WC, and urine iodine. WC, waist circumference. ${ }^{\dagger}$ GRS and 4 SNPs were not included in logistic regression analysis simultaneously.

\section{Relationship of 4 SNPs with TPOAb Positivity}

Logistic regression analysis indicated that genotype GG of rs9277555 and genotype TT of rs11675434 were associated with an increased risk of TPOAb positivity $(\mathrm{OR}=1.64$ and $1.57,5-95 \%$ CI 1.09, 2.47 and 1.01, 2.43, $p=0.02$ and 0.048 ). Genotype CC of rs301799 was a minor allele in Chinese population, while genotype TT did not increase the risk of TPOAb positivity. There was also no significant difference between rs11675434, rs3094228 and TPOAb positivity. Besides that, $G$ allele of rs 9277555 was significantly associated with TPOAb positivity $(\mathrm{OR}=$ $1.27,5-95 \%$ CI 1.04, 1.55, $p=0.02$ ) (Table 2).

\section{Association of Different Risk Factors with TPOAb Positivity}

In logistic regression analysis, TPOAb positivity was associated with rs9277555 (OR $=1.30,5-95 \%$ CI 1.05 , $1.61, p=0.02)$ and sex (OR $=2.53,5-95 \%$ CI 1.71, 3.74, $p<0.001$ ). Moreover, rs 9277555 was also significantly associated with $\mathrm{TPOAb}$ positivity $(\mathrm{OR}=1.30,5-95 \% \mathrm{CI}$ $1.05,1.61, p=0.02)$. Whereas other 3 SNPs showed no association with TPOAb positivity. TPOAb-GRS was significantly associated with TPOAb positivity $(\mathrm{OR}=$ $5.09,5-95 \%$ CI 1.30, 19.91, $p=0.02$ ) after adjusting for 
Fig. 2. Association of urine iodine and TPOAb positivity stratified by TPOAbGRS. a Prevalence of TPOAb positivity in different TPOAb-GRS quartiles. According to urine iodine level, each GRS group was divided into four quartiles, respectively. There was no significant difference between different urine iodine quartiles. b Higher number of TPOAb risk SNPs was not associated with increased TPOAb positivity.

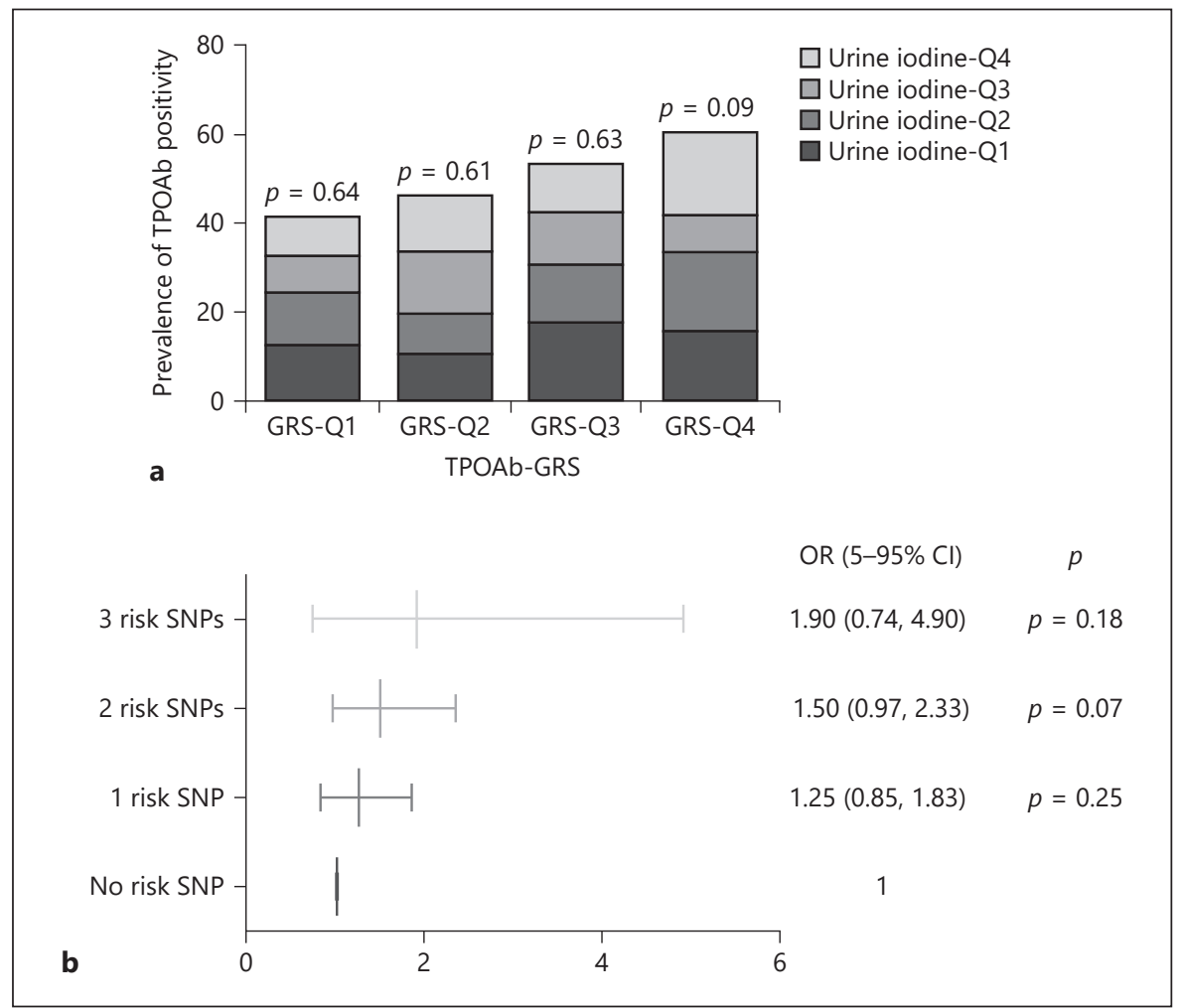

Table 4. TPOAb-GRS and the risk of TPOAb positivity

\begin{tabular}{llll}
\hline $\begin{array}{l}\text { TPOAb-GRS } \\
\text { quartile }\end{array}$ & $\begin{array}{l}\text { TPOAb-positivity, \% } \\
\text { (cases/total) }\end{array}$ & OR (5-95\% CI) & $p$ \\
\hline 1 (reference) & $10.8(61 / 565)$ & 1 & - \\
2 & $12.0(42 / 350)$ & $1.13(0.74,1.71)$ & 0.58 \\
3 & $13.5(53 / 394)$ & $1.28(0.87,1.90)$ & 0.21 \\
4 & $15.6(66 / 424)$ & $1.52(1.05,2.21)$ & 0.03 \\
\hline
\end{tabular}

sex, age, smoking, WC, and urine iodine (Table 3). Subjects with a high TPOAb-GRS had a 52\% increased risk of TPOAb positivity compared to subjects with a low TPOAb-GRS (OR 1.52, 5-95\% CI 1.05, 2.21, $p=0.03$ ) (Table 4).

\section{Interaction Effect on TPOAb Positivity}

TPOAb-GRS did not modify the association of urine iodine, smoking, and sex with TPOAb positivity ( $p$ for interaction $=0.76,0.32$, and 0.31 , respectively) (Table 3 ). There was no significant difference in the prevalence of TPOAb positivity among four quartiles of urine iodine in different TPOAb-GRS ( $p=0.64,0.61,0.63$, and 0.09) (Fig. 2a). With the increase of risk SNPs, there was no significant increase in $\mathrm{TPOAb}$ positivity $(\mathrm{OR}=1.25$,
5-95\% CI 0.85, 1.83, $p=0.25$; OR $=1.50,5-95 \%$ CI 0.97, $2.33, p=0.07 ; \mathrm{OR}=1.90,5-95 \%$ CI $0.74,4.90, p=0.18$ ) (Fig. 2b).

\section{Discussion}

In our study, we found that genotype GG of rs 9277555 (HLA-DPB1) was associated with an increased risk of TPOAb positivity $(\mathrm{OR}=1.64,5-95 \%$ CI 1.09, 2.47, $p=$ 0.02), whereas in Caucasian population, rs11675434 (TPO) was most significantly associated with TPOAb positivity [1], which only showed marginal increased risk of TPOAb positivity in our study. Logistic regression analysis showed TPOAb-GRS and rs9277555 were associated with TPOAb positivity $(\mathrm{OR}=5.09,5-95 \% \mathrm{CI} 1.30$, 19.91, $p=0.02$ and $\mathrm{OR}=1.30,5-95 \%$ CI 1.05, 1.61, $p=$ 0.02 ). Subjects with a high TPOAb-GRS had a $52 \%$ increased risk of TPOAb positivity compared to subjects with a low TPOAb-GRS. According to a GWAS metaanalysis, Medici et al. [2] calculated TPOAb-GRS based on five SNPs (rs11675434, rs653178, rs10944479, rs1230666, rs2010099). Subjects with a high GRS had 2.2 times increased risk of TPOAb positivity than those with a low GRS [2]. Moreover, rs10944479, rs653178, and 
rs1230666 had only one allele in Chinese population. However, they were significantly associated with TPOAb positivity in Western countries. So, susceptibility genes were different between Eastern and Western populations.

According to twin study data, the heritability of developing TPOAbs is approximately 70\% [5], but the identified risk loci for AITD only accounts for a minor proportion of the heritability, which indicates important effects of other factors, such as excessive iodine intake, selenium deficiency, bacterial or viral infection, and vitamin $\mathrm{D}$ deficiency [17].

In adults, the recommended daily iodine intake is 150 $\mu \mathrm{g}$, while in pregnant or in lactating women, the recommended daily iodine intake is $250 \mu \mathrm{g}$ [18]. Iodine deficiency causes several diseases, such as goiter and cretinism [19], which can be prevented with iodized salt. Over the past 60 years, China has experienced four stages in the implementation of universal salt iodization [20], including a nonmandatory salt iodization program, a mandatory universal salt iodization implemented throughout China, a national mandate for salt iodization, and a national decree for salt iodization. Now, China is identified as a country with more than adequate iodine intake by the WHO [6]. However, mandatory salt iodination resulted in an increased prevalence of AITD, while a decrease in iodine intake causes a decrease in the incidence of AITD [21]. Xu et al. [22] found that excess iodine suppressed autophagy and promoted apoptosis of thyroid follicular cells and is thus associated with Hashimoto thyroiditis. Iodine excess also increases intrathyroid-infiltrating Th 17 cells and inhibits $\mathrm{T}$ regulatory cell development, which triggers an abnormal expression of tumor necrosis factorrelated apoptosis-inducing ligand in thyrocytes, thus inducing apoptosis and parenchymal destruction [23].

Shan et al. [9] performed a large cross-sectional study to investigate the correlation of iodine status with thyroid disorder. Results showed the prevalence of positive thyroid antibodies were higher in cities with more than adequate iodine intake than in cities with adequate iodine intake (12.4 vs. $10.9 \%, p=0.004)$ [8]. Moreover, in Denmark, thyroid autoimmune antibodies are more common in iodine-adequate areas than in iodine-deficient areas [24], which indicates that excessive iodine intake might be an important environment factor in the pathogenesis of positive thyroid autoantibodies. However, Chen et al. [11] found low urine iodine concentrations might be a risk factor for AITD represented by TPOAb or TGAb positivity in Shanghai, which is an adequate iodine intake area. Recently, Sun et al. [25] also found that iodine deficiency during early pregnancy is an independent risk factor for both TPOAb positivity (OR $=1.64,5-95 \%$ CI $1.29-2.08)$ and TGAb positivity $(\mathrm{OR}=1.44,5-95 \% \mathrm{CI} 1.16-1.80)$. So the relationship between iodine intake and TPOAb positivity is very complex. Moreover, the interaction between genetic polymorphism and iodine intake in predisposing for TPOAb positivity was still unknown in the Chinese population. In our study, regression analysis found sex and rs9277555 were associated with TPOAb positivity. However, urine iodine was not associated with TPOAb positivity. So in the contribution of TPOAb positivity, we postulated that genetic factors might be more important than iodine intake in people living in Shanghai.

In our previous study, Chen et al. [11] found urine iodine concentration was significant higher in TPOAb or TGAb positivity group. Although the medium urine iodine in TPOAb-positive group is lower than negative group, there was no significant difference in these groups in our study. It might be caused by different group definition. Actually, we intended to study the interaction of TPOAb susceptibility genes and iodine intake. So different groups were divided by TPOAb positivity alone and there was no significant difference between two groups. In addition, relatively small sample in our study might also be a possible reason.

Our study had some strengths. First, there were many studies focused on iodine intake and TPOAb positivity. This is the first to investigate interaction between genetic polymorphisms and iodine intake in Chinese population. Second, data collection and measurement were performed by the same research group with strong quality control. However, our study also has some limitations. First, there was small sample in our study. So we cannot exclude bias in genotype allele distribution. Second, all the subjects came from Huangpu district in Shanghai, which is an adequate iodine intake area. Our results on gene-environment relationship were largely based on the adequate iodine intake. Third, thyroid dysfunction was self-reported. So we cannot exclude a very small amount of people with unconfirmed Graves' disease or Hashimoto thyroiditis. Fourth, there was significant difference in smoking and age between 280 subjects without urine iodine data and finally included 1,733 subjects. So, there was information bias in age and smoking.

In conclusion, TPOAb-GRS was associated with increased risk of TPOAb positivity in a Chinese Han population. However, there were no GRS-urine iodine interactions in our cohort. Functional studies still need to determine the relationship between SNPs and TPOAb-positive status. Finally, other environmental factors that contribute to the development of TPOAb positivity also need to be clarified.
Han/Chen/Chen/Wang/Yu/Cheng/Chen/ $\mathrm{Zhu} / \mathrm{Lu}$ 


\section{Statement of Ethics}

This study was carried out in accordance with the recommendations of the institutional ethical committee of the Shanghai Ninth People's Hospital affiliated with Shanghai Jiaotong University School of Medicine, with written informed consent from all subjects, and in accordance with the Declaration of Helsinki. The protocol was approved by the institutional ethical committee of the Shanghai Ninth People's Hospital affiliated with Shanghai Jiaotong University School of Medicine.

\section{Disclosure Statement}

The authors declare that they have no conflicts of interest to disclose.

\section{Funding Sources}

This study was supported by National Natural Science Foundation of China (91857117, 81670717); Science and Technology Commission of Shanghai Municipality (19140902400, 18410722300); the Major Science and Technology Innovation Program of Shanghai Municipal Education Commission (2019-01-0700-01-E00059); Commission of Health and Family Planning of Pudong District (PWZxq2017-17); Municipal Human Resources Development Program for Outstanding Young Talents in Medical and Health Sciences in Shanghai (2017YQ053); Shanghai JiaoTong University School of Medicine (19XJ11007).

\section{Author Contributions}

Y.L. and B.H. designed and supervised this investigation. B.H. and C.C. performed this investigation. Y.C., N.W., and J.Y. contributed to the data collection. J.C., Y.C., and C.Z. provided technical or material support. All authors read and approved the final manuscript.

\section{References}

1 Schultheiss UT, Teumer A, Medici M, Li Y, Daya N, Chaker L, et al. A genetic risk score for thyroid peroxidase antibodies associates with clinical thyroid disease in communitybased populations. J Clin Endocrinol Metab. 2015 May; 100(5):E799-807.

2 Medici M, Porcu E, Pistis G, Teumer A, Brown SJ, Jensen RA, et al. Identification of novel genetic Loci associated with thyroid peroxidase antibodies and clinical thyroid disease.PLoS Genet.2014Feb;10(2):e1004123.

3 Pearce EN, Farwell AP, Braverman LE. Thyroiditis. N Engl J Med. 2003 Jun;348(26):2646-55.

4 Schweizer U, Chiu J, Köhrle J. Peroxides and peroxide-degrading enzymes in the thyroid. Antioxid Redox Signal. 2008 Sep;10(9):1577-92.

5 Brix TH, Kyvik KO, Hegedüs L. A population-based study of chronic autoimmune hypothyroidism in Danish twins. J Clin Endocrinol Metab. 2000 Feb;85(2):536-9.

6 Hansen PS, Brix TH, Iachine I, Kyvik KO, Hegedüs $\mathrm{L}$. The relative importance of genetic and environmental effects for the early stages of thyroid autoimmunity: a study of healthy Danish twins. Eur J Endocrinol. 2006 Jan; 154(1):29-38.

7 Zimmermann MB, Andersson M. Update on iodine status worldwide. Curr Opin Endocrinol Diabetes Obes. 2012 Oct;19(5):382-7.

8 Liu P, Su XH, Shen HM, et al. National iodine deficiency disorders: an analysis of surveillance data in 2011. Zhonghua Difangbingxue Zazhi. 2015;34(3):181-5.

9 Shan Z, Chen L, Lian X, Liu C, Shi B, Shi L, et al. Iodine Status and Prevalence of Thyroid Disorders After Introduction of Mandatory Universal Salt Iodization for 16 Years in China: A Cross-Sectional Study in 10 Cities. Thyroid. 2016 Aug;26(8):1125-30.
10 Han B, Wang X, Wang N, Li Q, Chen Y, Zhu $\mathrm{C}$, et al. Investigation of vitamin $\mathrm{D}$ status and its correlation with insulin resistance in a Chinese population. Public Health Nutr. 2017 Jun;20(9):1602-8.

11 Chen C, Xu H, Chen Y, Chen Y, Li Q, Hu J, et al Iodized Salt Intake and Its Association with Urinary Iodine, Thyroid Peroxidase Antibodies, and Thyroglobulin Antibodies Among Urban Chinese. Thyroid. 2017 Dec;27(12):1566-73.

12 Xu Y, Wang L, He J, Bi Y, Li M, Wang T, et al.; 2010 China Noncommunicable Disease Surveillance Group. Prevalence and control of diabetes in Chinese adults. JAMA. 2013 Sep;310(9):948-59.

13 Bi Y, Wang W, Xu M, Wang T, Lu J, Xu Y, et al. Diabetes genetic risk score modifies effect of bisphenol A exposure on deterioration in glucose metabolism. J Clin Endocrinol Metab. 2016 Jan;101(1):143-50.

14 Pedersen IB, Knudsen N, Jørgensen T, Perrild $\mathrm{H}$, Ovesen L, Laurberg P. Thyroid peroxidase and thyroglobulin autoantibodies in a large survey of populations with mild and moderate iodine deficiency. Clin Endocrinol (Oxf). 2003 Jan;58(1):36-42.

15 Zhang Y, Shi L, Zhang Q, Peng N, Chen L, Lian $\mathrm{X}$, et al. The association between cigarette smoking and serum thyroid stimulating hormone, thyroid peroxidase antibodies and thyroglobulin antibodies levels in Chinese residents: A cross-sectional study in 10 cities. PLoS One. 2019 Nov; 14(11):e0225435.

16 Chen Y, Zhu C, Chen Y, Wang N, Li Q, Han B, et al. Are Thyroid Autoimmune Diseases Associated with Cardiometabolic Risks in a Population with Normal Thyroid-Stimulating Hormone? Mediators Inflamm. 2018 Oct; 2018:1856137.
17 Simmonds MJ. GWAS in autoimmune thyroid disease: redefining our understanding of pathogenesis. Nat Rev Endocrinol. 2013 May; 9(5):277-87.

18 Zhao F, Feng J, Li J, Zhao L, Liu Y, Chen H, et al. Alterations of the Gut Microbiota in Hashimoto's Thyroiditis Patients. Thyroid. 2018 Feb;28(2):175-86.

19 Zimmermann MB, Boelaert K. Iodine deficiency and thyroid disorders. Lancet Diabetes Endocrinol. 2015 Apr;3(4):286-95.

20 Pearce EN, Andersson M, Zimmermann MB Global iodine nutrition: where do we stand in 2013? Thyroid. 2013 May;23(5):523-8.

21 Miranda DM, Massom JN, Catarino RM, Santos RT, Toyoda SS, Marone MM, et al. Impact of nutritional iodine optimization on rates of thyroid hypoechogenicity and autoimmune thyroiditis: a cross-sectional, comparative study. Thyroid. 2015 Jan;25(1):118-24.

22 Xu C, Wu F, Mao C, Wang X, Zheng T, Bu L et al. Excess iodine promotes apoptosis of thyroid follicular epithelial cells by inducing autophagy suppression and is associated with Hashimoto thyroiditis disease. J Autoimmun. 2016 Dec;75:50-7.

23 Duntas LH. The role of iodine and selenium in autoimmune thyroiditis. Horm Metab Res. 2015 Sep;47(10):721-6.

24 Effraimidis G, Wiersinga WM. Mechanisms in endocrinology: autoimmune thyroid disease: old and new players. Eur J Endocrinol. 2014 Jun;170(6):R241-52.

25 Sun J, Teng D, Li C, Peng S, Mao J, Wang W, et al. Association between iodine intake and thyroid autoantibodies: a cross-sectional study of 7073 early pregnant women in an iodine-adequate region. J Endocrinol Invest. 2019 Jul.
Relationship between Gene

Polymorphisms and Urine Iodine
Eur Thyroid J 2021;10:79-85 DOI: $10.1159 / 000506701$ 